\title{
Neuroprotective effects of $\alpha$-iso-cubebene against glutamate- induced damage in the HT22 hippocampal neuronal cell line
}

\author{
SUN YOUNG PARK ${ }^{1}$, WON JUNG JUNG ${ }^{2}$, JUM SOON KANG ${ }^{2}$, CHEOL-MIN KIM $^{3}$, \\ GEUNTAE PARK $^{1,4}$ and YOUNG-WHAN CHOI ${ }^{2,5}$ \\ ${ }^{1}$ Bio-IT Fusion Technology Research Institute, Pusan National University, Busan 609-735; \\ ${ }^{2}$ Department of Horticultural Bioscience, Pusan National University, Miryang 627-706; \\ ${ }^{3}$ Research Center for Anti-Aging Technology Development, and ${ }^{4}$ Institute for Research and Industry Cooperation, \\ Pusan National University, Busan 609-735; ${ }^{5}$ Life and Industry Convergence Research Institute, \\ Pusan National University, Miryang 627-706, Republic of Korea
}

Received June 19, 2014; Accepted December 4, 2014

DOI: $10.3892 /$ ijmm.2014.2031

\begin{abstract}
Since oxidative stress is critically involved in excitotoxic damage, we sought to determine whether the activation of the transcription factors, cAMP-responsive element binding protein (CREB) and nuclear factor (erythroid-derived 2)-like 2 (Nrf2, also known as NFE2L2), by $\alpha$-iso-cubebene is involved in its protective effects against glutamate-induced neuronal cell death. Pre-treatment with $\alpha$-iso-cubebene significantly attenuated glutamate-induced cytotoxicity in mouse hippocampus-derived neuronal cells. $\alpha$-iso-cubebene also reduced the glutamate-induced generation of reactive oxygen species and calcium influx, thus preventing apoptotic cell death. $\alpha$-iso-cubebene inhibited glutamate-induced mitochondrial membrane depolarization and, consequently, inhibited the release of the apoptosis-inducing factor from the mitochondria. Immunoblot anlaysis revealed that the phosphorylation of extracellular signal-regulated kinase (ERK) by glutamate was reduced in the presence of $\alpha$-iso-cubebene. $\alpha$-iso-cubebene activated protein kinase A (PKA), CREB and Nrf2, which mediate the expression of the antioxidant enzymes, heme oxygenase-1 (HO-1) and $\mathrm{NAD}(\mathrm{P}) \mathrm{H}$ dehydrogenase [quinone] 1 (NQO1), involved in neuroprotection. In addition, $\alpha$-iso-cubebene induced the expression of antioxidant responsive element and CRE transcriptional activity, thus conferring neuroprotection against glutamate-induced oxidative injury. $\alpha$-iso-cubebene also induced the expression of Nrf2-dependent genes encoding HO-1 and NQO1. Furthermore, the knockdown of CREB and Nrf2 by small interfering RNA attenuated the neuroprotective effects of $\alpha$-iso-cubebene. Taken together, our results indicate that $\alpha$-iso-cubebene protects HT22 cells from glutamate-induced
\end{abstract}

Correspondence to: Professor Young-Whan Choi, Department of Horticultural Bioscience, Pusan National University, 50 Cheonghak-ri, Samnangjin-eup, Miryang, Gyeongnam 627-706, Republic of Korea E-mail: ywchoi@pusan.ac.kr

Key words: $\alpha$-iso-cubebene, glutamate, apoptosis, cAMP-responsive element binding protein, Nrf2, neuroprotection oxidative damage through the activation of Nrf2/HO-1/NQO-1, as well as through the PKA and CREB signaling pathways.

\section{Introduction}

Neuronal cytotoxicity occurs when there is an imbalance in the systems that generate and scavenge reactive oxygen species (ROS) in a number of pathological processes, including neurodegenerative diseases $(1,2)$. Although glutamate is an important neurotransmitter in mammals, high concentrations of this amino acid can contribute to neuronal cell death. Moreover, glutamate toxicity induces the progressive death of neuronal cells and this has been implicated in a variety of neurological disorders, such as Parkinson's disease, Alzheimer's disease, seizures, ischemia and trauma $(3,4)$. Glutamate has been shown to induce neuronal cell death through two mechanisms: glutamate receptor-induced cytotoxicity and ROS-mediated oxidative stress. HT22 mouse hippocampal neuronal cells are a useful model for studying the mechanisms of glutamateinduced oxidative stress which leads to cell death. Since this cell line lacks functional ionotropic glutamate receptors, the excessive accumulation of glutamate causes oxidative injury due to the suppression of the cellular uptake of cysteine through the cysteine/glutamate transport system; this, in turn, causes a progressive depletion of glutathione (GSH), an intracellular antioxidant molecule $(5,6)$.

In addition, the excessive production of ROS induces the activation of mitogen-activated protein kinase (MAPK) signaling pathways, which, in turn, induces the transcription of numerous apoptosis-related genes. MAPKs are serine/threonine protein kinases involved in cell proliferation and differentiation, as well as in inflammation, cancer and apoptosis. MAPKs can be subdivided into three classes based on sequence homology: the extracellular signal-regulated kinase (ERK), Jun N-terminal kinases (JNKs) and the p38 kinases. MAPKs can be phosphorylated and then activated in response to a variety of extracellular stimuli, leading to the expression or activation of key molecules. Recent studies have shown that ERK is also responsible for oxidative stress in neurodegenerative disorders, ischemia and stroke (7-9). There may be a close association between excessive 
ROS production and MAPK activation in glutamate-induced neuronal cell death. Additionally, glutamate toxicity is caused by increased intracellular calcium influx due to GSH depletion, but not through the glutamate receptor. Previous studies have demonstrated that glutamate promotes an increase in calcium influx in HT22 cells, and acts as a key regulator in response to oxidative stress in neuronal cells $(10,11)$.

Furthermore, glutamate-induced oxidative stress, ROS production and calcium influx are crucial contributors to mitochondrial dysfunction $(12,13)$. Apoptosis-inducing factor (AIF) is released from the mitochondria and translocates to the nucleus in its truncated form (tAIF), promoting the transcription of apoptosis-related genes. Therefore, the inhibition of the apoptotic pathway activated by glutamate-induced oxidative stress may be an excellent strategy for the prevention or treatment of neurodegenerative disorders (14).

To counteract glutamate-induced oxidative stress insults, neuronal cells have evolved defense mechanisms, such as antioxidant enzymes. With the induction of antioxidant enzymes, such as heme oxygenase-1 (HO-1) and $\mathrm{NAD}(\mathrm{P}) \mathrm{H}$ dehydrogenase [quinone] 1 (NQO1), neuronal cells are more resistant to the subsequent provocation of glutamate-induced stress. The importance of HO-1 and NQO1 expression in mediating neuroprotective properties has been well characterized in neurodegenerative disorders $(15,16)$. HO-1 and NQO1 expression is regulated through transcription factors, such as nuclear factor (erythroid-derived 2)-like 2 (Nrf2, also known as NFE2L2), cAMP-responsive element binding protein (CREB) and activating protein-1 (AP-1). The promoter regions of the HO-1 and NQO1 genes have antioxidant responsive elements (AREs) to which Nrf2 can directly bind. The activation of Nrf2 by various stimulators is followed by its translocation to the nucleus, where it modulates the expression of ARE-driven genes (such as HO-1 and NQO1). Protein kinase A (PKA)-mediated CREB activation also induces the expression of these ARE-driven genes (HO-1 and NQO1). CREB activation mediates the transcription of genes containing a cAMP-responsive element. Previous studies have indicated that the PKA/CREB/Nrf2 signaling pathway regulates the expression of the ARE-driven genes (HO-1 and NQO1) and has subsequent neuroprotective effects (17-20).

We previously isolated a novel natural compound, $\alpha$-isocubebene, from Schisandra chinensis, an herb used for food, tea and wine production, as well as in Traditional Chinese Medicine (23). Recently, we published a study on the beneficial bioactivities of $\alpha$-iso-cubebene, such as its anti-inflammatory, antiseptic and immunomodulatory activities (24). Moreover, recent studies have suggested that $\alpha$-iso-cubebene inhibits amyloid $\beta$-induced inflammation in microglia (21-23). However, the mechanisms underlying its potential protective effects on glutamate-induced neuronal cell death have not yet been fully investigated. In this study, we investigated the neuroprotective effects of $\alpha$-iso-cubebene against glutamate-induced neuronal cell death using mouse hippocampus-derived neuronal cells (HT22 cells). Our results demonstrated that $\alpha$-iso-cubebene attenuated glutamate-induced cell death through the conservation of mitochondrial function in HT22 cells. The data from our study suggest that the expression of HO-1 and NQO1 may be at least partially responsible for the neuroprotective effects of $\alpha$-iso-cubebene, which involves the activation of the Nrf2 and PKA/CREB signaling pathways.

\section{Materials and methods}

Reagents. Dulbecco's modified Eagle's medium (DMEM) and fetal bovine serum (FBS) were purchased from Gibco-BRL (Grand Island, NY, USA). 3-[4,5-Dimethythiazol-2-yl]-2,5diphenyltetrazolium bromide (MTT) and other reagents were purchased from Sigma-Aldrich (St. Louis, MO, USA). Small interfering RNA (siRNA) against CREB and Nrf2, and antibodies against $\alpha$-tubulin (sc-23948), TATA-binding protein (TBP; sc-204), ERK (sc-94), JNK (sc-571), p38 (sc-7149), Nrf2 (sc-722), HO-1 (sc-10789) and NQO1 (sc-16464) were purchased from Santa Cruz Biotechnology, Inc. (Santa Cruz, CA, USA). Antibodies against AIF (4642), COX IV (4850), phosphorylated (p-)ERK (4370), p-JNK (4671), p-p38 (9215), p-PKA (4781), PKA (4782), p-CREB (9198) and CREB (9197) were purchased from Cell Signaling Technology (Beverly, MA, USA). The FuGENE HD transfection reagent and the X-tremeGENE siRNA Transfection reagent were obtained from Roche Diagnostics (Indianapolis, IN, USA). The cytotoxicity detection kit [lactate dehydrogenase (LDH) assay] was purchased from Roche Applied Science (Rotkreuz, Switzerland). The APO-BrdU ${ }^{\mathrm{TM}}$ TUNEL Assay kit was purchased from Invitrogen (Carlsbad, CA, USA). The nuclear extraction kit was purchased from Active Motif (San Diego, CA, USA). The mitochondria Isolation kit was purchased from Thermo Scientific (Rockford, IL, USA).

Cell culture. The HT22 hippocampal cell line was obtained from Professor Youn-Chul Kim, Wonkwang University (Iksan, Korea). The cells were grown as monolayers in DMEM supplemented with 5\% heat-inactivated FBS. The cells were incubated at $37^{\circ} \mathrm{C}$ in a humidified atmosphere containing $5 \% \mathrm{CO}_{2}$. To avoid changes in cell characteristics caused by extended periods of cell culture, all experiments were conducted with cells between passages 15 and 25 . Each cell suspension was subcultured by treatment with trypsin/EDTA every 2 days in order to maintain exponential growth.

Cell viability assay. The cells were incubated in wells of a 24-well plate at a density of $4 \times 10^{4}$ cells/well. MTT solution $(50 \mu \mathrm{g} / \mathrm{ml})$ was added to each well. The plates were then incubated for an additional $4 \mathrm{~h}$ at $37^{\circ} \mathrm{C}$ in a $5 \% \mathrm{CO}_{2}$ atmosphere, after which the supernatant was removed. Formazan crystals that had formed in viable cells were solubilized using dimethyl sulfoxide (DMSO). The absorbance of each well was measured at $570 \mathrm{~nm}$ by using a microplate reader (Wallace, Boston, MA, USA).

LDH release assay. Extracellular LDH activity was spectrophotometrically measured using a cytotoxicity detection kit according to the manufacturer's instructions. Briefly, the cells were seeded in 96-well plates and then subjected to the indicated treatments. For analysis, the supernatant was extracted from each well, and catalyst solutions were added followed by incubation for $30 \mathrm{~min}$ at room temperature. The absorbance of each well was measured at $490 \mathrm{~nm}$ using a microplate reader (Wallace).

Measurement of intracellular ROS and calcium levels. To evaluate the levels of intracellular ROS and calcium, the cells were treated with $\mathrm{CM}-\mathrm{H}_{2} \mathrm{DCFDA}$ (an indicator of ROS production) or Fluo-4-AM (an indicator of general oxidative stress; both 
from Invitrogen), for $1 \mathrm{~h}$ at $37^{\circ} \mathrm{C}$ under $5 \% \mathrm{CO}_{2}$. The cells were then harvested and washed 3 times with phosphate-buffered saline (PBS). The fluorescence intensity was then measured by flow cytometry (using the flow cytometer at the Bio-IT Fusion Technology Research Institute, Pusan National University, Busan, Korea) at an excitation wavelength of $488 \mathrm{~nm}$ and an emission wavelength of $525 \mathrm{~nm}$. Data analyses were performed using CXP software 2.0 (Beckman Coulter, Brea, CA, USA).

Measurement of sub-G1 cell population. The cells were collected by centrifugation at $800 \mathrm{rpm}$ for $3 \mathrm{~min}$ after treatment, washed twice in PBS, and fixed with $75 \%$ ethanol overnight. Prior to flow cytometric analysis, the fixed cells were washed with PBS and incubated with a final concentration of $50 \mu \mathrm{g} /$ $\mathrm{ml}$ propidium iodide for $10 \mathrm{~min}$ in the dark. The percentage of apoptotic cells was calculated as the percentage of the sub-G1 peak as determined using CXP software 2.0 (Beckman Coulter).

Terminal deoxynucleotidyl transferase-mediated dUTP nick end labeling (TUNEL) assay. The DNA ladder pattern in the cells was evaluated by TUNEL assay using an APO-BrdU ${ }^{\text {Tм }}$ TUNEL Assay kit (Invitrogen) according to the manufacturer's instructions. The results were analyzed by flow cytometry (fluorescein isothiocyanate; excitation at $488 \mathrm{~nm}$ and emission at $520 \mathrm{~nm}$ ). Data analyses were performed using CXP 2.0 (Beckman Coulter).

Assay for mitochondrial membrane potential (MMP, $\left.\Delta \psi_{m}\right)$. $\operatorname{MMP}\left(\Delta \psi_{\mathrm{m}}\right)$ was determined by flow cytometry using the J-aggregate forming lipophilic cationic probe, 5,5',6,6'-tetrachloro-1,1',3,3'-tetraethyl-benzimidazolylcarbocyanine iodide (JC-1). The cells were stained with JC-1 and analyzed by flow cytometry with CXP 2.0 analysis software (Beckman Coulter). JC-1 red fluorescence, indicating an intact $\Delta \psi \mathrm{m}$, was excited at $488 \mathrm{~nm}$, and emission was detected using a $613 \pm 20 \mathrm{~nm}$ bandpass filter. For each sample, 10,000 cells were acquired and analyzed by flow cytometry. Data were analyzed using the fluorescence intensity of the analyzed cell population.

Protein extracts and western blot analysis. The cytosol, nuclear and mitochondrial extracts were isolated with a nuclear extraction kit according to the manufacturer's instructions. The protein content of the cell lysates was then determined using the Bradford protein assay (Bio-Rad, Hercules, CA, USA). The protein in each sample was resolved by sodium dodecyl sulfate-polyacrylamide gel electrophoresis, transferred onto a polyvinylidene difluoride membrane, and exposed to the appropriate antibody. The proteins were visualized by an enhanced chemiluminescence detection system (Amersham Biosciences, Piscataway, NJ, USA) using horseradish peroxidase-conjugated anti-rabbit or anti-mouse secondary antibody. Images were acquired using an ImageQuant 350 analyzer (Amersham Biosciences).

Transient transfection with siRNA. Transfection of the cells with siRNA was performed using the X-tremeGENE siRNA Transfection Reagent (Roche Applied Science) according to manufacturer's instructions. Commercially available mouse CREB- and Nrf2-specific siRNAs and negative control siRNAs (both from Santa Cruz, Heidelberg, Germany) were used for transfection. Briefly, X-tremeGENE siRNA transfection reagent (10 $\mu \mathrm{l}$ ) was added to $100 \mu \mathrm{l}$ of serum-free medium containing
$2 \mu \mathrm{g}$ of each siRNA followed by incubation for $20 \mathrm{~min}$ at room temperature. Gene silencing was measured after $48 \mathrm{~h}$ by western blot analysis.

Transient transfection and dual luciferase assay. The cells were transfected with the CRE and ARE-reporter plasmid, or an HO-1 promoter reporter plasmid using FuGENE-HD reagent according to manufacturer's instructions. A Renilla luciferase control plasmid, pRL-CMV, was co-transfected as an internal control for transfection efficiency. Luciferase activity was assayed using a dual-luciferase assay kit according to manufacturer's instructions. Luminescence was measured using a microplate luminometer (Wallac 1420).

Statistical analysis. Data are expressed as the means \pm standard error (SE). Statistical analysis was performed using the Statistical Package for the Social Sciences (SPSS) software (version 18.0) to identify significant differences based on either one- or two-way analysis of variance (ANOVA) followed by Dunn's post-hoc tests. P-values $<0.05$ were considered to indicate statistically significant differences. Each experiment was repeated at least 3 times.

\section{Results}

Inhibitory effects of $\alpha$-iso-cubebene on the glutamate-induced death of HT22 cells. To examine the ability of $\alpha$-iso-cubebene to exert neuronal protective effects against glutamate-induced cell death, its effects on glutamate-induced cell death on the murine hippocampal neuronal cell line, HT22, were investigated. To accomplish this, the HT22 cells were first pre-treated with non-cytotoxic concentrations of $\alpha$-iso-cubebene [isolated from Schisandra chinensis $(20-80 \mu \mathrm{M})]$ for $12 \mathrm{~h}$, and then treated with glutamate for $12 \mathrm{~h}$. Cell viability was then measured by MTT assay. Cell viability was recovered by $\alpha$-iso-cubebene in a dose-dependent manner (Fig. 1A). The neuroprotective effects of $\alpha$-iso-cubebene were also verified by $\mathrm{LDH}$ release assay. The results revealed that glutamate significantly increased LDH release from the HT22 cells. However, the cells treated with $\alpha$-iso-cubebene prior to glutamate incubation displayed a dosedependent attenuation of LDH release (Fig. 1B).

It is fairly well established that glutamate toxicity is mediated by ROS production and oxidative stress-induced cell death (25). Hence, in the current study, we investigated whether the neuroprotective effects of $\alpha$-iso-cubebene involved the blockage of glutamate-induced oxidative stress in hippocampal neuronal cells. Cellular oxidative stress was determined by $\mathrm{H}_{2}$ DCF-DA, based on the ROS-mediated conversion of $\mathrm{H}_{2} \mathrm{DCF}-\mathrm{DA}$ to fluorescent DCF. Glutamate increased intracellular ROS production in the HT22 cells. By contrast, at the tested concentrations, $\alpha$-iso-cubebene profoundly reduced the glutamate-induced production of ROS (Fig. 1C). In addition, glutamate-mediated cytotoxicity results in GSH depletion through the suppression of cysteine uptake and increased intracellular calcium concentration. Abnormal levels of GSH and calcium in cells consequently induce ROS overproduction (13). Therefore, in this study, in order to determine the association between the protective effects of $\alpha$-iso-cubebene on neuronal cells and glutamate-induced cell death, its inhibitory activity against glutamate-induced calcium influx was examined. Calcium influx in HT22 cells was signifi- 

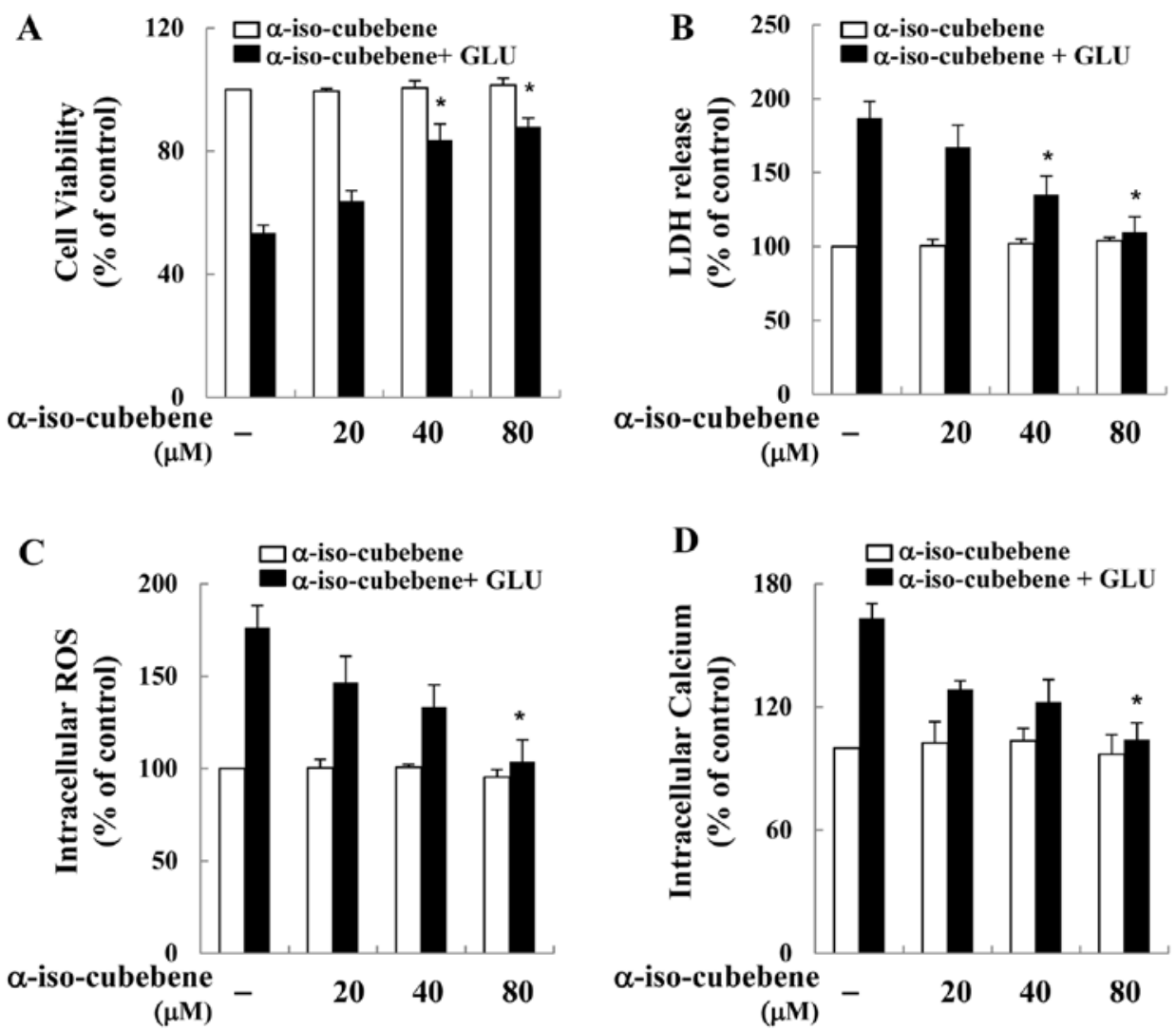

Figure 1. Effects of $\alpha$-iso-cubebene on glutamate-induced cell death, intracellular reactive oxygen species (ROS) production and calcium accumulation. HT22 hippocampal cells were treated with various concentrations of $\alpha$-iso-cubebene for $12 \mathrm{~h}$, followed by glutamate $(5 \mathrm{mM})$ treatment for $12 \mathrm{~h}$. Cell viability was determined by (A) MTT assay or a (B) lactate dehydrogenase (LDH) release assay. (C) Intracellular ROS levels were detected by $\mathrm{H}_{2}$ DCFDA using flow cytometry. (D) Intracellular calcium was assessed by Fluo-4-AM using flow cytometry. Each bar represents the mean \pm standard error (SE) from 3 independent experiments per group. ${ }^{*} \mathrm{P}<0.05$ vs. the glutamate-treated group.

cantly reduced by $\alpha$-iso-cubebene treatment, whereas that of the glutamate-treated cells was increased compared to the controls (untreated cells; Fig. 1D).

$\alpha$-iso-cubebene attenuates glutamate-induced apoptosis in HT22 cells. It has been suggested that excessive glutamate levels results in apoptosis (12). Thus, in this study, in order to investigate the role of $\alpha$-iso-cubebene in glutamate-induced apoptosis in HT22 cells, the cells were treated with glutamate for the indicated periods of time, and the effects of $\alpha$-iso-cubebene on cell cycle distribution were analyzed. As demonstrated by our results, the ratio of sub-G1 cells was increased following treatment with glutamate, indicating an increase in glutamate-induced apoptosis. However, glutamate-induced apoptosis was decreased in the $\alpha$-iso-cubebene-pre-treated cells compared to the controls (untreated cells; Fig. 2A). Additionally, to verify the specific type of cell death elicited by glutamate, TUNEL assay was performed to assess the typical DNA ladder pattern of the glutamate-treated HT22 cells. The HT22 cells that had undergone $24 \mathrm{~h}$ of glutamate treatment showed an increase in apoptosis compared to the controls, whereas pre-treatment with $\alpha$-iso-cubebene resulted in a significant decrease in cell apoptosis (Fig. 2B).

$\alpha$-iso-cubebene modulates glutamate-induced mitochondrial function and AIF localization. Mitochondrial membrane disruption is a primary process in apoptosis; therefore, the JC-1 stain was used to detect the glutamate-induced changes in MMP. With JC-1 staining, the HT22 cells treated with glutamate displayed a significantly increased green fluorescence intensity compared to the controls (untreated cells), indicating the glutamate-induced disruption of MMP $\left(\Delta \psi_{\mathrm{m}}\right)$. By contrast, pre-treatment with $\alpha$-iso-cubebene reversed the glutamate-induced increase in green fluorescence (Fig. 3A). Thus, mitochondrial function was effectively protected by treatment with $\alpha$-iso-cubebene.

Apoptosis correlates with the levels of proteins that regulate mitochondrial-dependent apoptosis and the apoptotic pathway induced by AIF signaling (14). As previously demonstrated, the disruption of MMP leads to the release of the pro-apoptotic factors, cytochrome $c$, AIF and endonuclease G (Endo G). Upon these changes, the normal form of AIF is cleaved and released from the mitochondria (14). In this study, to determine the effects of $\alpha$-iso-cubebene on AIF distribution, mitochondrial, cytosolic and nuclear fractions of AIF were analyzed by western blot analysis. COX IV is specific to the inner mitochondrial membrane protein, $\alpha$-tubulin is specific to the cytoplasmic protein and TBP is specific to the nuclear fraction. COX IV, $\alpha$-tubulin and TBP are commonly used for determining the mitochondrial, cytosolic and nuclear fractions in western blot analysis. Following treatment with glutatame, the level of AIF in the mitochondrial fractions was decreased at the indicated time points (Fig. 3B). By contrast, treatment with glutamate increased the cytosolic and nuclear fractions of AIF after $12 \mathrm{~h}$, and treatment with 
A
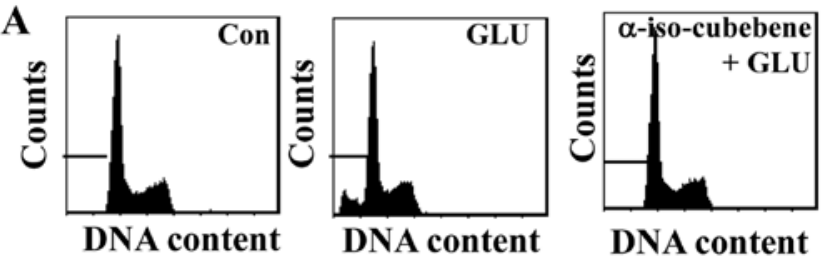

DNA content

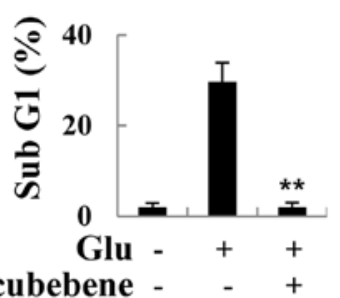

$\alpha$-iso-cubebene - - +

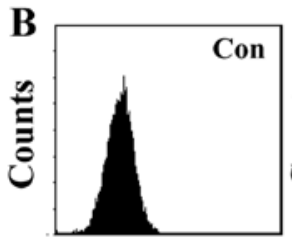

Fluorescence

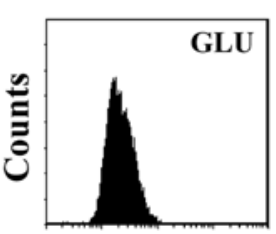

Fluorescence
Fluorescence
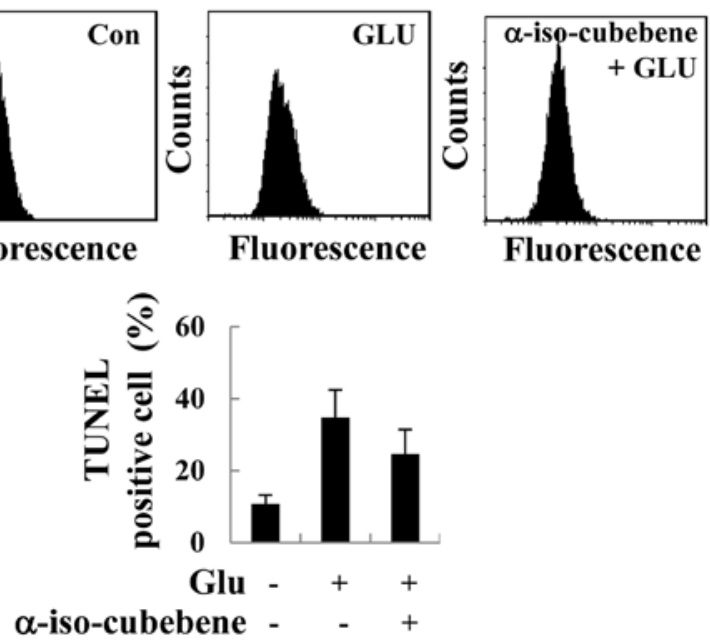

Figure 2. Effects of $\alpha$-iso-cubebene on glutamate-induced apoptotic cell death. Cells were pre-treated with $\alpha$-iso-cubebene for $12 \mathrm{~h}$, followed by glutamate $(5 \mathrm{mM})$ treatment for $12 \mathrm{~h}$. (A) The sub-G1 peak was determined by flow cytometry. Cell death was determined by propidium iodide staining, followed by flow cytometry. Quantitative analysis of the histograms expressed as the percentage of apoptotic HT22 cells observed in the same field. (B) Cell apoptosis was measured by terminal deoxynucleotidyl transferase-mediated dUTP nick end labeling (TUNEL) assay. TUNEL-stained cells were quantified by flow cytometry. The number in each panel is the percentage of TUNELpositive cells. Quantitative analysis of the histograms expressed as the ratio of TUNEL-positive HT22 cells observed in the same field. Each bar represents the mean \pm standard error (SE) from 3 independent experiments per group. ${ }^{*} \mathrm{P}<0.05$ vs. the glutamate-treated group.

$\alpha$-iso-cubebene reversed the glutamate-induced translocation of AIF at this time point (Fig. 3B). Thus, these data suggest that $\alpha$-iso-cubebene effectively suppresses the glutamate-induced disruption of MMP and the translocation of AIF.

$\alpha$-iso-cubebene suppresses the activation of ERK induced by glutamate. Emerging evidence indicates that MAPK activation and oxidative stress are involved in the pathophysiology of neurodegenerative diseases. MAPK activation and oxidative stress also induce apoptosis in neuronal cells (26). Therefore, the effects of $\alpha$-iso-cubebene on the glutamate-mediated activation of ERK, JNK and p38 MAPKs were examined. The levels of p-ERK, p-p38 and p-JNK were increased after 4-8 h of glutamate treatment (Fig. 4). However, the levels of p-ERK were significantly reduced following treatment with $\alpha$-iso-cubebene.
$\mathbf{A}$
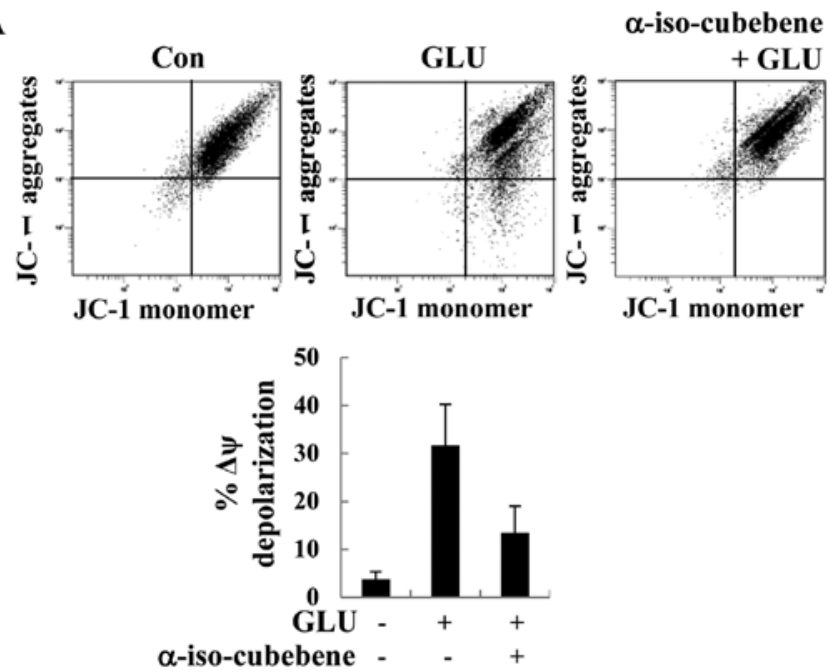

B

\section{GLU}

$\alpha$-iso-cubebene - $\quad-\quad-\quad-\quad-\quad-\quad+\quad+++$

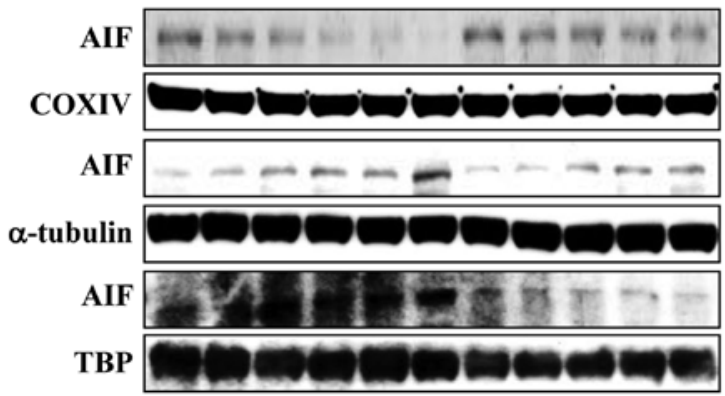

Figure 3. Effects of $\alpha$-iso-cubebene on mitochondrial membrane potentials (MMP, $\Delta \psi \mathrm{m}$ ) and apoptosis-inducing factor (AIF) translocation. Cells were treated with $\alpha$-iso-cubebene $(20 \mu \mathrm{M})$ for $12 \mathrm{~h}$ and then incubated with glutamate $(5 \mathrm{mM}$ ) for $12 \mathrm{~h}$. (A) Cells were then stained with $\mathrm{JC}-1$ dye probe and analyzed by flow cytometry. Numbers in lower right quadrant indicate the percentage of cells that emit only green fluorescence, which is attributed to depolarized mitochondrial membrane. (B) Cells were incubated with $\alpha$-iso-cubebene $(20 \mu \mathrm{M})$ for $12 \mathrm{~h}$, and then stimulated with glutamate $(5 \mathrm{mM})$ for the indicated periods of time; translocation of AIF was analyzed by western blot analysis.

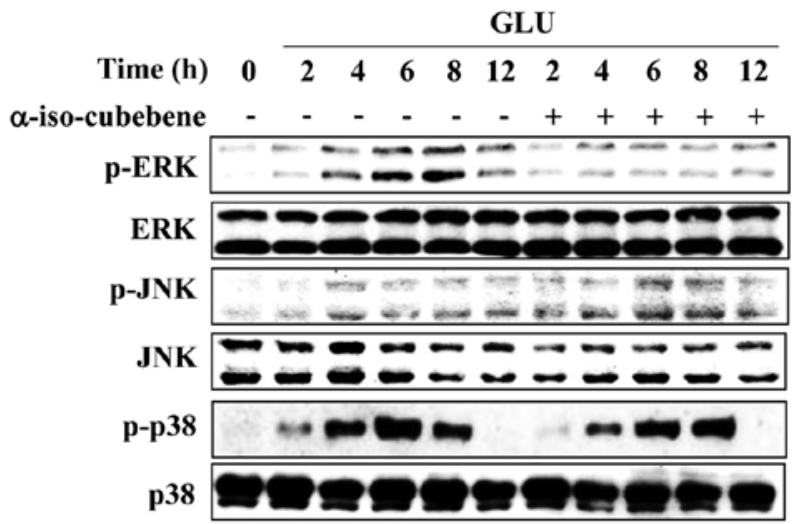

Figure 4. Effects of $\alpha$-iso-cubebene on the glutamate-induced phosphorylation of extracellular signal-regulated kinase (ERK), Jun N-terminal kinase (JNK), and p38 mitogen-activated protein kinases (MAPKs). Cells were incubated with $\alpha$-iso-cubebene $(20 \mu \mathrm{M})$ for $12 \mathrm{~h}$, and then stimulated with glutamate $(5 \mathrm{mM})$ for the indicated periods of itme. Following incubation, the cells were harvested and ERK, JNK and p38 MAPKs levels were measured by western blot analysis using phospho-specific antibodies for ERK, JNK and p38 MAPKs. 
A

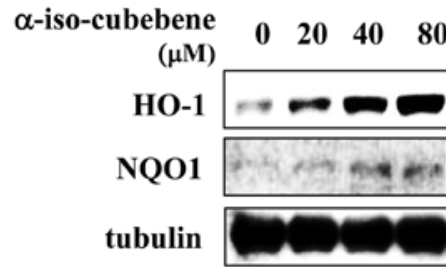

C

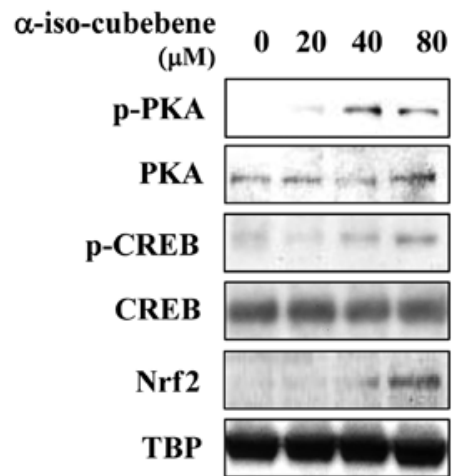

B

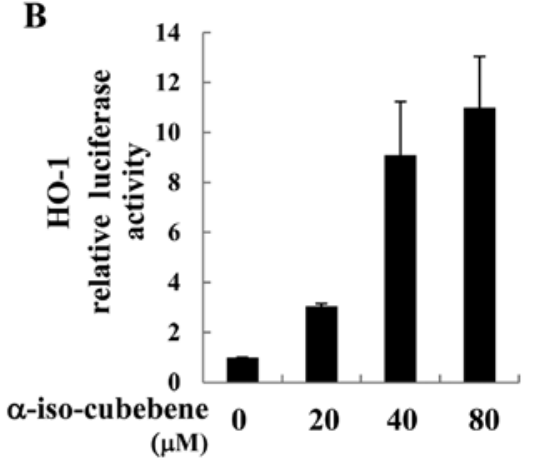

D

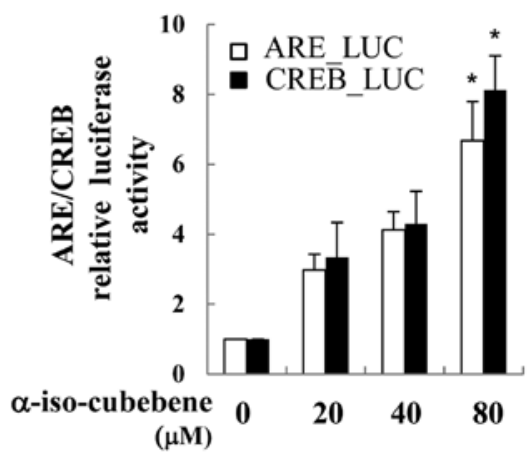

Figure 5. Effects of $\alpha$-iso-cubebene on the protein kinase A (PKA)/cAMP-responsive element binding protein (CREB) and nuclear factor (erythroidderived 2)-like 2 (Nrf2, also known as NFE2L2) activation and heme oxygenase-1 (HO-1) and NAD(P)H dehydrogenase [quinone] 1 (NQO1) expression. (A) Cells were cultured with $\alpha$-iso-cubebene for $12 \mathrm{~h}$, after which HO-1 and NQO-1 expression were determined by western blot anlaysis. (B) Cells were transfected with HO-1 promoter-luciferase construct and equal amounts of cell extracts were assayed for dual luciferase activity. (C) Cells were incubated with $\alpha$-iso-cubebene for $4 \mathrm{~h}$, and p-PKA, PKA, p-CREB and CREB expression and nuclear localization of Nrf2 was determined by western blot analysis. (D) Cells were transfected with the antioxidant responsive element (ARE) or CRE-luciferase construct then treated with $\alpha$-iso-cubebene. Equal amounts of cell extract were assayed for dual-luciferase activity. Expression of the Renilla luciferase control was used to normalize ARE or CRE-luciferase activity. Each bar represents the mean \pm standard error (SE) from 3 independent experiments per group. ${ }^{*} \mathrm{P}<0.05$ vs. the control-treated group.

$\alpha$-iso-cubebene-induced activation of PKA/CREB/Nrf2 and the expression of $\mathrm{HO}-1$ and NQO1. Previous data have indicated a critical role of ARE-driven genes in the host defense against a variety of physiological insults, such as oxidative stress, hypoxia and pro-inflammatory cytokines, as well as in the regulation of normal immune function (15). In this study, $\alpha$-iso-cubebene increased ARE-driven gene (HO-1 and NQO1) expression in a time and dose-dependent manner. The HT22 cells treated with $\alpha$-iso-cubebene had increased expression levels of HO-1 and NQO1 (Fig. 5A). To determine the effects of $\alpha$-iso-cubebene on HO- 1 expression, the HT22 cells were transiently transfected with HO-1 promoter reporter plasmid. Treatment with $\alpha$-iso-cubebene increased HO-1 promoter activity in a dose-dependent manner (Fig. 5B). Transcription factors that ARE-driven genes are associated with CREB and Nrf2 (27). Therefore, we investigated whether $\alpha$-iso-cubebene enhances CREB and Nrf2 activation. The evaluation of the phosphorylation status of PKA and CREB revealed that the maximum levels of their activation occurred $4 \mathrm{~h}$ following treatment with $\alpha$-iso-cubebene (data not shown). $\alpha$-isocubebene increased PKA and CREB phosphorylation, thereby affecting the total levels of PKA and CREB. In addition to CREB and Nrf2 activation, we examined the downstream effects of $\alpha$-iso-cubebene on Nrf2 activation. To accomplish this, we investigated the $\alpha$-iso-cubebene-induced Nrf2 nuclear translocation in HT22 cells (Fig. 5C). To elucidate the mecha- nisms underlying these effects, we used a luciferase reporter gene driven by Nrf2-binding to ARE and CRE-binding to CRE to analyze Nrf2 and CREB transactivity. The results revealed that $\alpha$-iso-cubebene significantly increased ARE and CRE transactivity in the HT22 cells (Fig. 5D).

Role of the CREB/Nrf2 pathway in the neuroprotective effects of $\alpha$-iso-cubebene. CREB and Nrf2 have been identified as key mediators in the regulation of the crosstalk between the neuroprotective signals induced by antioxidant gene cell response (28). In this study, we confirmed that $\alpha$-iso-cubebene markedly inhibited glutamate-induced cell death and that this effect correlated with the activation of the CREB and Nrf2 pathways. For these experiments, we used CREB and Nrf2 siRNA systems to silence the CREB and Nrf2 proteins, and then determined the effects on LDH release and performed TUNEL assay in HT22 cells. The HT22 cells were transfected with control siRNA or CREB siRNA or Nrf2 siRNA for $48 \mathrm{~h}$. Western blot analysis of the treated cells revealed that CREB and Nrf2 siRNA caused significantly knocked down CREB and Nrf2 by $48 \mathrm{~h}$ compared to the control siRNA-transfected cells (data not shown). The HT22 cells were transfected with CREB and Nrf2 siRNA, followed by treatment with $\alpha$-iso-cubebene and glutamate. The inhibitory effects of $\alpha$-iso-cubebene on the glutamate-induced release of LDH and the number of TUNEL-positive cells were abolished by CREB and Nrf2 siRNA, suggesting the involvement of the 
A

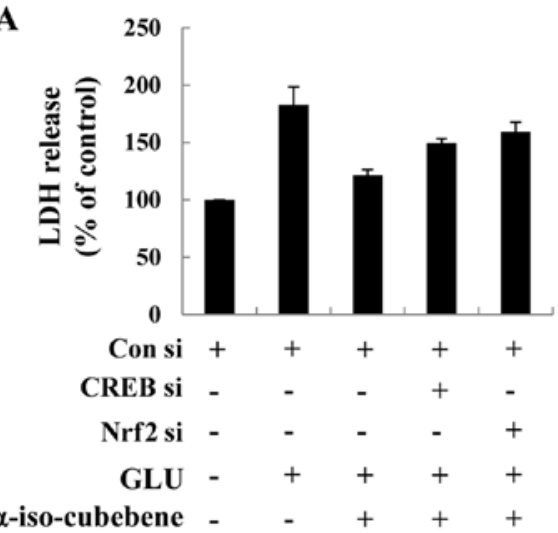

B

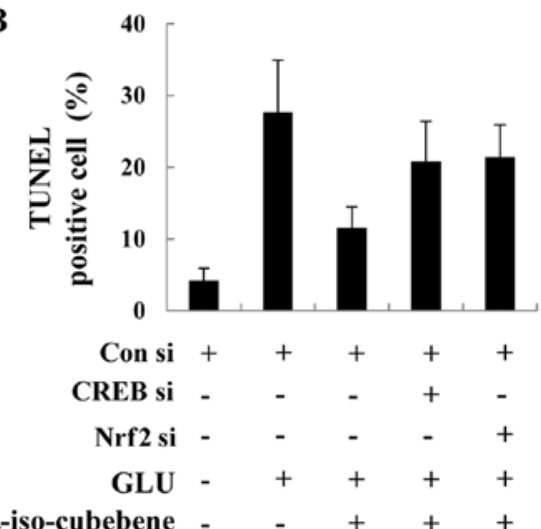

Figure 6. Effects of cAMP-responsive element binding protein (CREB) and nuclear factor (erythroid-derived 2)-like 2 (Nrf2, also known as NFE2L2) disruption on the $\alpha$-iso-cubebene anti-apoptotic activity in glutamate-stimulated HT22 cells. Cells were transfected with control, CREB, or Nrf2 siRNA using the $\mathrm{X}$-tremeGENE reagent according to the manufacturer's instructions. The cells were treated with $20 \mu \mathrm{M} \alpha$-iso-cubebene for $12 \mathrm{~h}$, and then stimulated with glutamate for $12 \mathrm{~h}$; after these treatments, they were subjected to (A) Lactate dehydrogenase (LDH) release assay and (B) terminal deoxynucleotidyl transferasemediated dUTP nick end labeling (TUNEL) assay.

PKA/CREB/Nrf2 pathway in the $\alpha$-iso-cubebene-induced neuroprotection in HT22 cells (Fig. 6).

\section{Discussion}

The results of this study demosntrated that $\alpha$-iso-cubebene significantly protected HT22 neuronal cells from glutamateinduced oxidative death by inhibiting the production of intracellular ROS and calcium. These findings support a neuroprotective role of $\alpha$-iso-cubebene through the PKA/CREB/Nrf2 signaling pathway and its downstream antioxidant enzymes. HT22, an immortalized mouse hippocampal neuronal cell line, has been used as an in vitro model of Alzheimer's disease, a disease characterized by neuronal cell death, particularly in the hippocampus. Moreover, the loss of neuronal cells due to oxidative stress appears to be a common cause contributing to neurodegenerative diseases $(29,30)$. Glutamate is a major contributor to neuronal cell death through oxidative toxicity in HT22 cells. The mechanisms of glutamate-induced toxicity in HT22 cells involve excessive glutamate levels, leading to the depletion of GSH, an intracellular antioxidant material, by disrupting the glutamate/cysteine antiporter system. Following GSH depletion, a series of biological processes occur, including ROS accumulation, calcium influx and lipid peroxidation (30). In this study, we assessed glutamate-induced HT22 cell death and neurotoxicity by MTT and LDH release assays and confirmed that $\alpha$-iso-cubebene markedly attenuated the glutamate-induced decrease in cell viability and the increase in LDH release. We also examined glutamate-induced apoptotic cell death in the HT22 cell line, which is typically characterized by hypodiploid DNA content and DNA fragmentation. These apoptotic features were detected by cell cycle analysis and TUNEL assays. Of note, treatment with $\alpha$-iso-cubebene markedly attenuated these apoptotic features, indicating that $\alpha$-iso-cubebene may be qualified for inhibiting glutamate-induced apoptotic cell death. We also examined the effects of $\alpha$-iso-cubebene on glutamateinduced cell death in HT22 cells by evaluating the inhibition of intracellular ROS and calcium accumulation. Since these events are known to play a major role in glutamate-induced neuronal cell death $(3,4)$, the inhibition of intracellular ROS and calcium influx in its early stages may be a central point for the $\alpha$-isocubebene-induced neuroprotective effects.

The disruption of MMP induced by oxidative stress is regulated by several anti- and pro-apoptotic proteins, such as Bcl-2 and Bax. During the apoptotic process, Bcl-2 and Bax change their location, allowing Bax to enter the outer mitochondrial membrane, permeabilizing it, and causing the release of cytochrome $c$ and the translocation of AIF. Mitochondrial apoptosis is divided into two types: caspase-dependent and caspase-independent. Caspase-independent apoptosis is mainly dependent on promoting the AIF translocation and disrupting the function and structure of the nucleus (14). Consistent with previous data (12), the present results suggest that glutamate-induced HT22 cell death occurs through mitochondrial dysfunction and the release of AIF from the mitochondria. However, $\alpha$-isocubebene prevented both glutamate-induced mitochondrial dysfunction and the abnormal distribution of AIF.

Protein phosphatases play an important role in controlling the magnitude and duration of MAPK activation (8). Thus, this study focused on the possible role of MAPK family members, which are known to play crucial roles during oxidative stress in many cell types $(7,8)$. Previous studies have suggested that the inhibition of ERK activation protects cells against glutamateinduced death $(7,8)$. In agreement with these studies, a previous study also found that glutamate led to the persistent activation of ERK, which was associated with the death of HT22 cells (8). Our results indicated that glutamate-induced oxidative toxicity led to the phosphorylation of MAPKs, including JNK, ERK, and p38, resulting in persistent neuronal cell death. By contrast, $\alpha$-iso-cubebene was able to significantly suppress the phosphorylation of ERK at the examined time points, albeit with little effect on JNK and p38 phosphorylation.

HO-1 and NQO1 are binding sites for the Nrf2 transcription factor and, as such, key signaling antioxidant enzymes for the regulation of neuroprotection through oxidative responses. A recent study demonstrated an inverse correlation between the antioxidant enzyme level and neuroprotection (31). In this study, we examined whether $\alpha$-iso-cubebene increases the expression of HO-1 and NQO1. Treatment with $\alpha$-iso-cubebene induced the expression of HO-1 and HO-1 in the HT22 cells. Additionally, 
a number of neuroprotective drugs have been shown to inhibit glutamate-induced neuronal cell death by stimulating the PKA/CREB/Nrf2 pathway $(20,32)$. In agreement with these studies, the results of the present study demonstrated that $\alpha$-iso-cubebene induced the activation of the PKA/CREB/Nrf2 pathway, thus providing a basis for the neuroprotective effects against glutamate-induced toxicity in HT22 cells.

In conclusion, $\alpha$-iso-cubebene was selected as a candidate for this fundamental research for investigating the neuroprotective drugs from natural products. $\alpha$-iso-cubebene exerted neuroprotective effects against glutamate-induced apoptosis in HT22 cells by suppressing the generation of ROS and calcium influx, as well as mitochondrial disruption, events thought to be involved in HT22 cell apoptosis. $\alpha$-iso-cubebene inhibited the glutamate-induced neuronal cell death by inducing HO-1 and NQO1 expression through the PKA/CREB/Nrf2 pathway. These data suggest that $\alpha$-iso-cubebene may have therapeutic potential as a neuroprotective drug for the treatment of neurodegenerative diseases.

\section{Acknowledgements}

This study was supported by the Basic Science Research Program through the National Research Foundation of Korea (NRF) funded by the Ministry of Education, Science and Technology (2012R1A1A3010601) and the Bio-industry Technology Development Program (311054-03-3-HD120), Ministry for Food, Agriculture, Forestry and Fisheries, Republic of Korea.

\section{References}

1. Coyle JT and Puttfarcken P: Oxidative stress, glutamate, and neurodegenerative disorders. Science 262: 689-695, 1993.

2. Tan S, Sagara Y, Liu Y, Maher P and Schubert D: The regulation of reactive oxygen species production during programmed cell death. J Cell Biol 141: 1423-1432, 1998

3. Elphick LM, Hawat M, Toms NJ, Meinander A, Mikhailov A, Eriksson JE and Kass GE: Opposing roles for caspase and calpain death proteases in L-glutamate-induced oxidative neurotoxicity. Toxicol Appl Pharmacol 232: 258-267, 2008

4. Murphy TH, Miyamoto M, Sastre A, Schnaar RL and Coyle JT: Glutamate toxicity in a neuronal cell line involves inhibition of cystine transport leading to oxidative stress. Neuron 2: 1547-1558, 1989.

5. Behl C, Lezoualc'h F, Trapp T, Widmann M, Skutella T and Holsboer F: Glucocorticoids enhance oxidative stress-induced cell death in hippocampal neurons in vitro. Endocrinology 138: 101-106, 1997.

6. Maher P and Schubert D: Signaling by reactive oxygen species in the nervous system. Cell Mol Life Sci 57: 1287-1305, 2000.

7. Stanciu M, Wang Y, Kentor R, et al: Persistent activation of ERK contributes to glutamate-induced oxidative toxicity in a neuronal cell line and primary cortical neuron cultures. J Biol Chem 275: 12200-12206, 2000.

8. Ravassard P, Pachoud B, Comte JC, et al: Paradoxical (REM) sleep deprivation causes a large and rapidly reversible decrease in long-term potentiation, synaptic transmission, glutamate receptor protein levels, and ERK/MAPK activation in the dorsal hippocampus. Sleep 32: 227-240, 2009.

9. Kumamaru E, Numakawa T, Adachi N, et al: Glucocorticoid prevents brain-derived neurotrophic factor-mediated maturation of synaptic function in developing hippocampal neurons through reduction in the activity of mitogen-activated protein kinase. Mol Endocrinol 22: 546-558, 2008

10. Fukui M, Song JH, Choi J, Choi HJ and Zhu BT: Mechanism of glutamate-induced neurotoxicity in HT22 mouse hippocampal cells. Eur J Pharmacol 617: 1-11, 2009.

11. Choi DW: Calcium-mediated neurotoxicity: Relationship to specific channel types and role in ischemic damage. Trends Neurosci 11: 465-469, 1988.
12. Ankarcrona M, Dypbukt JM, Bonfoco E, Zhivotovsky B, Orrenius S, Lipton SA and Nicotera P: Glutamate-induced neuronal death: a succession of necrosis or apoptosis depending on mitochondrial function. Neuron 15: 961-973, 1995.

13. Gunter TE and Pfeiffer DR: Mechanisms by which mitochondria transport calcium. Am J Physiol 258: C755-C786, 1990.

14. Zhang Y and Bhavnani BR: Glutamate-induced apoptosis in neuronal cells is mediated via caspase-dependent and independent mechanisms involving calpain and caspase- 3 proteases as well as apoptosis inducing factor (AIF) and this process is inhibited by equine estrogens. BMC Neurosci 7: 49, 2006.

15. Calkins MJ, Johnson DA, Townsend JA, et al: The Nrf2/ARE pathway as a potential therapeutic target in neurodegenerative disease. Antioxid Redox Signal 11: 497-508, 2009.

16. van Muiswinkel FL and Kuiperij HB: The Nrf2-ARE signalling pathway: promising drug target to combat oxidative stress in neurodegenerative disorders. Curr Drug Targets CNS Neurol Disord 4: 267-281, 2005.

17. Satoh T, McKercher SR and Lipton SA: Nrf2/ARE-mediated antioxidant actions of pro-electrophilic drugs. Free Radic Biol Med 65: 645-657, 2013.

18. Lee J, Kim CH, Simon DK, et al: Mitochondrial cyclic AMP response element-binding protein (CREB) mediates mitochondrial gene expression and neuronal survival. J Biol Chem 280: 40398-40401, 2005.

19. Baxter PS, Martel MA, McMahon A, Kind PC and Hardingham GE: Pituitary adenylate cyclase-activating peptide induces long-lasting neuroprotection through the induction of activity-dependent signaling via the cyclic AMP response element-binding protein-regulated transcription co-activator 1 . J Neurochem 118: 365-378, 2011.

20. Park SY, Kim JH, Lee SJ and Kim Y: Involvement of PKA and HO-1 signaling in anti-inflammatory effects of surfactin in BV-2 microglial cells. Toxicol Appl Pharmacol 268: 68-78, 2013.

21. Choi YW, Kim HJ, Park SS, et al: Inhibition of endothelial cell adhesion by the new anti-inflammatory agent alpha-isocubebene. Vascul Pharmacol 51: 215-224, 2009.

22. Lee SK, Kim SD, Lee HY, et al: $\alpha$-Iso-cubebene, a natural compound isolated from Schisandra chinensis fruit, has therapeutic benefit against polymicrobial sepsis. Biochem Biophys Res Commun 426: 226-231, 2012.

23. Lee YJ, Shim JW, Lee YJ, et al: Identification of a novel compound that stimulates intracellular calcium increase and CXCL8 production in human neutrophils from Schisandra chinensis. Biochem Biophys Res Commun 379: 928-932, 2009.

24. Park SY, Park SJ, Park NJ, Joo WH, Lee SJ and Choi YW: $\alpha$-Iso-cubebene exerts neuroprotective effects in amyloid beta stimulated microglia activation. Neurosci Lett 555: 143-148, 2013.

25. Maher P and Davis JB: The role of monoamine metabolism in oxidative glutamate toxicity. J Neurosci 16: 6394-6401, 1996.

26. Ziady AG, Sokolow A, Shank S, Corey D, et al: Interaction with CREB binding protein modulates the activities of Nrf2 and NF- $\kappa \mathrm{B}$ in cystic fibrosis airway epithelial cells. Am J Physiol Lung Cell Mol Physiol 302: L1221-L1231, 2012.

27. Wang T, Gu J, Wu PF, et al: Protection by tetrahydroxystilbene glucoside against cerebral ischemia: Involvement of JNK, SIRT1, and NF-kappaB pathways and inhibition of intracellular ROS/RNS generation. Free Radic Biol Med 47: 229-240, 2009.

28. Katoh Y, Itoh K, Yoshida E, Miyagishi M, Fukamizu A and Yamamoto M: Two domains of Nrf2 cooperatively bind CBP, a CREB binding protein, and synergistically activate transcription. Genes Cells 6: 857-868, 2001.

29. Arundine M and Tymianski M: Molecular mechanisms of glutamate-dependent neurodegeneration in ischemia and traumatic brain injury. Cell Mol Life Sci 61: 657-668, 2004.

30. Tofighi R, Johansson C, Goldoni M, Ibrahim WN, Gogvadze V, Mutti A and Ceccatelli S: Hippocampal neurons exposed to the environmental contaminants methylmercury and polychlorinated biphenyls undergo cell death via parallel activation of calpains and lysosomal proteases. Neurotox Res 19: 183-194, 2011.

31. Zhang H, Liu H, Davies KJ, Sioutas C, Finch CE, Morgan TE and Forman HJ: Nrf2-regulated phase II enzymes are induced by chronic ambient nanoparticle exposure in young mice with agerelated impairments. Free Radic Biol Med 52: 2038-2046, 2012.

32. Wang H, Guo Z, Wu F, et al: PKA-mediated protein phosphorylation protects ezrin from calpain I cleavage. Biochem Biophys Res Commun 333: 496-501, 2005. 